\title{
Use of coronary computed tomography angiography in the diagnosis and/or exclusion of coronary disease - Initial experience of one center and determination of the local acuity of the method, in comparison with coronary angiography
}

Pedro PG ${ }^{1 *}$, Carôla B ${ }^{1}$, Conduto $\mathrm{R}^{1}$, Oliveira $\mathrm{P}^{1}$, Pereira $\mathrm{M}^{1}$, Morais $\mathrm{J}^{1}$, Costa J ${ }^{1}$, Silva $\mathrm{C}^{2}$, Barão $\mathrm{I}^{1}$, Vieira $\mathrm{E}^{1}$, Magalhães $\mathrm{MP}^{1}$ and Ferreira RC ${ }^{1}$ ${ }^{1}$ Hospital SAMS-PICS, Lisbon, Portugal

${ }^{2}$ School of Health Technology of Lisbon (ESTeSL) and Statistics and Applications Center of the University of Lisbon (CEAUL), Portugal

\begin{abstract}
Coronary computed tomography angiography (CCTA) is becoming the primary method for diagnosing/excluding coronary disease (CD), particularly in patients with a low to intermediate probability. Its sensitivity $(\mathrm{Ss})$, specificity $(\mathrm{Sp})$ and predictive $(\mathrm{PV})$ value are well known. However, local diagnostic acuity of the method is not always tested but should be known by each center. We started a CCTA program and describe our experience with a 64-detector, single-source scanner. We studied the first 700 consecutive patients, aged $64 \pm 10$ years, $64 \%$ men, with a BMI of $27 \pm 4$. The technical quality of the study was good in 609 patients $(87 \%)$, fair in 84 patients (12\%) and poor in 7 patients (1\%). In 42 of the patients, a subsequent invasive coronary angiography (ICA) was necessary. In this group, the two methods were correlated. The time difference between the two examinations was $35 \pm 28$ days. A total of 168 coronary arteries were analyzed (only native and non-stented arteries). Coronary stenosis $\geq 50 \%$ in the main trunk and $\geq 70 \%$ in the other arteries (LAD, Cx and RCA) were regarded as significant lesions, by both methods on a semi-quantitative basis. $\mathrm{Ss}, \mathrm{Sp},+\mathrm{PV}$ and -PV results of $91 \%, 80 \%, 83 \%$ and $89 \%$ by patient and $83 \%, 93 \%, 74 \%$ and $95 \%$ by artery, were respectively obtained.

Conclusion: CCTA proved to be a simple and effective, method for assessing coronary anatomy. In our study, conducted under local conditions, we achieved a diagnostic capacity similar to that described in the main reference studies. This assessment is regarded as vital, contributing to the credibility of the technique used in each center.
\end{abstract}

\section{Introduction}

Coronary computed tomography angiography (CCTA) is gradually becoming the primary method for diagnosing or excluding coronary artery disease, particularly in patients with a low to intermediate probability, in a wide variety of clinical contexts. The sensitivity, specificity and positive and negative predictive value of this technique are well known, having been published in several singleand multi-center studies [1-3]. However, these correlation studies do not always reflect the local day-to-day situation, which is why it is extremely important that each center initiates a CCTA program and tests the usability of the examination as well as its diagnostic acuity under local conditions and daily clinical practice. This was what we proposed to do.

\section{Objectives}

Our proposed objectives were to: 1 - Analyze the clinical indications for CCTA; 2 - Assess the pre-test probability of coronary disease (where applicable) in the population studied; 3 - Describe the study protocol; 4 - Assess the radiation doses used; 5 - Assess the quality of the study; 6 - Compare the results with invasive coronary angiography in a selected group of patients who underwent both examinations.

\section{Material and methods}

\section{Type of study}

This was a consecutive, observational, prospective study of patients referred for CCTA.

\section{Clinical indications and pre-test probability of coronary disease}

The indications for the study were arranged into seven main groups: Group 1: Chest pain to be explained and/or ischemia tests with alterations. Group 2: Risk stratification with calcium scoring. Group 3: Assessment of coronary artery bypasses. Group 4: Assessment of coronary stents. Group 5:

Assessment of the thoracic aorta. Group 6: Exclusion of myocardial coronary disease/heart valve

diseases. Group 7: Other situations.

Correspondence to: Paulo G Pedro, Hospital SAMS-PICS, Lisbon, Portugal. E-mail: paulo.pedro@sams.sbsi.pt

Key words: coronary computed tomography angiography, coronary disease, angiographic correlation

Received: March 20, 2018; Accepted: March 25, 2018; Published: March 30, 2018 
Pedro PG (2018) Use of coronary computed tomography angiography in the diagnosis and/or exclusion of coronary disease - Initial experience of one center and determination of the local acuity of the method, in comparison with coronary angiography

The pre-test probability of coronary disease was assessed using the Diamond Forrester [4] tables, according to age, sex and type of precordial pain complaint (Table 1).

\section{Equipment used and study protocol}

A SOMATOM Perspective ${ }^{\star}$ CT scanner (Siemens AG) was used, which had the following technical characteristics: single source: 110$130 \mathrm{kV}$; collimation: 64 detectors $\times 0.6 \mathrm{~mm}$ slice; spatial resolution - $0.35 \mathrm{~mm}$; temporal resolution - 165 to $195 \mathrm{~ms}$ (due to the type of protocol used); iterative reconstruction $\left(\mathrm{SAFIRE}^{\circledR}\right)$, and other low-dose algorithms (Care Dose $\mathrm{kV}^{\oplus}$ and ECG pulse ${ }^{\oplus}$ ). The target heart rate (HR) was less than $60 \mathrm{bpm}$, with oral and/or intravenous beta-blockers being freely used, unless contraindicated. To ensure acquisition during the lowest and most stable HR possible, the apnea method following a double breath was used. The contrast agent used was iopromide 370 at a flow rate of $6 \mathrm{cc} / \mathrm{s}$, preferably through a straight $18 \mathrm{G}$ antecubital catheter, followed by the same volume and flow rate of saline solution (double bolus). All the patients were given $0.5 \mathrm{mg}$ of sublingual nitroglycerin, unless contraindicated or if only calcium scoring was carried out. The sequential or spiral acquisition protocol was determined using the ECG Check $^{\circledast}$ tool, which, by analyzing the HR and its stability, recommended a protocol through a semaphore-type suggestion (green - recommended protocol; yellow - recommended with reservations, and red - protocol not recommended). After acquisition, the images were reconstructed for calcium scoring in $3 \mathrm{~mm}$ slices of the best diastole and for the CCTA in $0.75 \mathrm{~mm}$ thin slices of the best diastole and best systole (chosen automatically by the equipment), in a highdefinition matrix of 512 lines. The left ventricular function was obtained (in the spiral studies) by reconstructing 10 phases of the cardiac cycle (every 10\%) in a matrix of 256 lines. Finally, the mediastinum and lung were reconstructed in $4 \mathrm{~mm}$ slices. Where necessary, additional reconstructions were carried out in other cardiac cycle phases to improve the image quality. These images were then post-processed and analyzed using the syngo.via ${ }^{\oplus}$ version VA11. Orthogonal and oblique two-dimensional reconstructions, curved plane reformatting (Figure 1), thin-slice maximum intensity projections (MIP) (Figure 1), threedimensional reconstructions (volume rendering technique - Figure 1) and AngioView $\omega^{\oplus}$ reconstructions (Figure 1) were carried out. The ventricular function, volumes and masses were assessed and the left ventricle was segmented according to the recommendations of the American Society of Echocardiography with 16 segments.

Table 1. Probability of coronary disease based on sex, age and type of symptoms

\begin{tabular}{|c|c|c|c|c|c|c|c|c|}
\hline \multirow[b]{2}{*}{ Age } & \multicolumn{2}{|c|}{ Asymptomatic } & \multicolumn{2}{|c|}{$\begin{array}{l}\text { Non-anginal } \\
\text { chest pain }\end{array}$} & \multicolumn{2}{|c|}{ Atypical angina } & \multicolumn{2}{|c|}{ Typical angina } \\
\hline & Men & Women & Men & Women & Men & Women & Men & Women \\
\hline $30-39$ & $1.9 \%$ & $0.3 \%$ & $5.2 \%$ & $0.8 \%$ & $21.8 \%$ & $4.2 \%$ & $69.7 \%$ & $25.8 \%$ \\
\hline $40-49$ & $5.5 \%$ & $1.0 \%$ & $14.1 \%$ & $2.8 \%$ & $46.1 \%$ & $13.3 \%$ & $87.3 \%$ & $55.2 \%$ \\
\hline $50-59$ & $9.7 \%$ & $3.2 \%$ & $21.5 \%$ & $8.4 \%$ & $59.9 \%$ & $32.4 \%$ & $92.0 \%$ & $79.4 \%$ \\
\hline $60-69$ & $12.3 \%$ & $7.5 \%$ & $28.1 \%$ & $18.6 \%$ & $67.1 \%$ & $54.4 \%$ & $94.3 \%$ & $90.6 \%$ \\
\hline
\end{tabular}
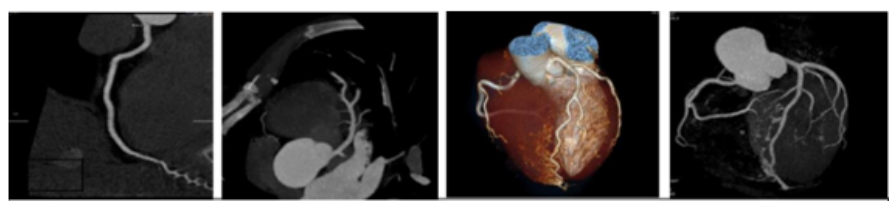

Figure 1: Coronary reconstructions: 1.1 Curved plane reconstruction (normal RCA). 1.2 Thin-slice maximum intensity projection (MIP) (normal LAD). 1.3 Volume rendering technique (VRT). 1.4 AngioView

\section{Radiation doses used}

The ALARA (As Low As Reasonably Achievable) principle was used. The total dose was measured as DLP (dose length product) and the constant of 0.014 was used as the millisievert conversion factor, by which the DLPs were multiplied. The first and second years of use of the equipment were separated in order to compare the total dose used.

\section{Assessment of the study quality}

The study quality was assessed subjectively by the operator according to the following general principle: Good quality - highquality display of all epicardial coronary segments (up to the vascular diameter of $1.5 \mathrm{~mm}$ ); Reasonable quality - presence of slight movement artifacts not significantly compromising the interpretation of the study; Deficient quality - presence of artifacts or poor vascular opacification not allowing diagnosis in arteries with a diameter in excess of $1.5 \mathrm{~mm}$.

\section{Assessment of the study safety}

All complications or adverse events recorded were assessed.

\section{Analysis and comparison of the coronary assessment results}

The coronary assessment results were analyzed according to the recommendations of the Society of Cardiovascular Computed Tomography, revised in 2014 [5]. A subjective classification was used, with those coronary stenoses equal to or exceeding $50 \%$ in the main trunk (MT) and equal to or exceeding $70 \%$ in the other coronary arteries (left anterior descending - LAD, circumflex - Cx, right coronary artery RCA) and respective branches being regarded as significant stenoses. The criteria used in the coronary angiography were the same (50\% in the main trunk and $70 \%$ in the other arteries). The ICA assessment was also conducted semi-quantitatively by two observers, with the final decision being made by a third observer, where necessary. The results were subsequently compared with those from the CCTA. The arteries that could not be interpreted due to artifacts were regarded as having significant coronary stenoses. Coronary artery bypasses and coronary arteries with stents were excluded from the comparison.

\section{Statistical analysis}

The means and standard deviation of the demographic variables of the population were analyzed. In the comparison of the CCTA and coronary angiography results, the sensitivity, specificity, and positive and negative predictive value were analyzed by a dichotomic variation (with or without disease) according to Bayes' theorem, and the ROC (receiver operating characteristics) curves were assessed to analyze the probability of disease. The IBM SPSS Statistics software, version 20.0, was used and the significance level was set at $5 \%$.

\section{Results}

From 6/13/2012 to $10 / 13 / 2014$ we studied 700 consecutive patients, aged $64 \pm 10$ years, with $64 \%$ men. The Body Mass Index (BMI) was $27 \pm 4$. The heart rate during acquisition was $55 \pm 8 \mathrm{bpm}$. The clinical indications are shown in Table 2 . The pre-test probability of coronary disease was $42 \pm 18 \%$.

The type of study carried out was as follows: calcium scoring only in $18 \%$ of patients; CCTA only in $14 \%$ of pts; calcium scoring and CCTA in $67 \%$ of patients. The CCTA protocol (according to the results suggested by ECG Check $^{\circledR}$ ) was as follows: Spiral - 90\%; Bi-segment spiral - $6 \%$; Sequential - $4 \%$. The average radiation dose (only taking into account studies with a "cardiac window", i.e. from the carina to the diaphragm) 
Pedro PG (2018) Use of coronary computed tomography angiography in the diagnosis and/or exclusion of coronary disease - Initial experience of one center and determination of the local acuity of the method, in comparison with coronary angiography

was $5.7 \mathrm{msV}$. Analyzed by year, in the first year this value was $7.05 \mathrm{msV}$ and in the second and subsequent years it was $4.96 \mathrm{msV}$. The study quality was deemed to be "good" in 609 patients (87\%), "reasonable" in 84 patients (12\%) and "deficient" in 7 patients (1\%).

A coronary angiography was carried out on 42 of the patients referred for a CCTA. The pre-test probability of coronary disease in this group was $52 \pm 18 \%$. The time difference between the two examinations was $35 \pm 28$ days, during which there was no change in the clinical status of patients.

A total of 168 coronary arteries were analyzed (only native and nonstented arteries). In six of these, stenoses could not be quantified due to CCTA artifacts. For comparison purposes, these cases were regarded by default as having significant coronary disease. The comparison between the two techniques according to the methodology described is shown in Table 3.

The ROC curve for the CCTA was estimated (Figure 2) and the area under the ROC curve was calculated $(\mathrm{AUC}=0.899)$, together with the respective $95 \%$ confidence interval $(\mathrm{CI}=0.789 ; 1.00)$. In order to assess the correlation between the CCTA and coronary angiography, the McNemar's test ( $\mathrm{p}$-value $=0.99)$ and the Kappa coefficient $(\mathrm{k}=0.798$, $\mathrm{p}$-value $<0.001$ ) were applied.

Table 2. Indications for CCTA

\begin{tabular}{|l|c|c|}
\hline Indication & Number & $\mathbf{\%}$ \\
\hline $\begin{array}{l}\text { Precordial pain to be explained and/or non-invasive tests } \\
\text { for suspected ischemia }\end{array}$ & 406 & $58 \%$ \\
\hline Risk stratification with calcium scoring & 128 & $18 \%$ \\
\hline Assessment of coronary artery bypasses & 78 & $11 \%$ \\
\hline Assessment of coronary stents & 28 & $4 \%$ \\
\hline Assessment of the aorta & 22 & $3 \%$ \\
\hline $\begin{array}{l}\text { Exclusion of myocardial coronary disease/heart valve } \\
\text { diseases }\end{array}$ & 22 & $3 \%$ \\
\hline Other & 16 & $2 \%$ \\
\hline
\end{tabular}

Table 3. Acuity of CCTA compared to coronary angiography by patient and by artery

\begin{tabular}{|l|c|c|c|c|c|}
\hline Acuity by patient & Angio + & Angio - & Total & Sensitivity & $91 \%$ \\
\hline CCTA + & 20 & 4 & 24 & Specificity & $80 \%$ \\
\hline CCTA - & 2 & 16 & 18 & $P P V$ & $83 \%$ \\
\hline Total & 22 & 20 & 42 & NPV & $89 \%$ \\
\hline Acuity by artery - all & Angio + & Angio - & Total & Sensitivity & $83 \%$ \\
\hline CCTA + & 29 & 10 & 39 & Specificity & $93 \%$ \\
\hline CCTA - & 6 & 123 & 129 & PPV & $74 \%$ \\
\hline Total & 35 & 133 & 168 & NPV & $95 \%$ \\
\hline Acuity by artery - LM & Angio + & Angio - & Total & Sensitivity & $100 \%$ \\
\hline CCTA + & 1 & 1 & 2 & Specificity & $98 \%$ \\
\hline CCTA - & 0 & 40 & 40 & PPV & $50 \%$ \\
\hline Total & 1 & 41 & 42 & NPV & $100 \%$ \\
\hline Acuity by artery - LAD & Angio + & Angio - & Total & Sensitivity & $91 \%$ \\
\hline CCTA + & 10 & 6 & 16 & Specificity & $81 \%$ \\
\hline CCTA - & 1 & 25 & 26 & PPV & $63 \%$ \\
\hline Total & 11 & 31 & 42 & NPV & $96 \%$ \\
\hline Acuity by artery - Cx & Angio + & Angio - & Total & Sensitivity & $73 \%$ \\
\hline CCTA + & 8 & 2 & 10 & Specificity & $94 \%$ \\
\hline CCTA - & 3 & 29 & 32 & PPV & $80 \%$ \\
\hline Total & 11 & 31 & 42 & NPV & $91 \%$ \\
\hline Acuity by artery - RCA & Angio + & Angio - & Total & Sensitivity & $83 \%$ \\
\hline CCTA + & 10 & 1 & 11 & Specificity & $97 \%$ \\
\hline CCTA - & 2 & 29 & 31 & PPV & $91 \%$ \\
\hline Total & 12 & 30 & 42 & NPV & $94 \%$ \\
\hline
\end{tabular}

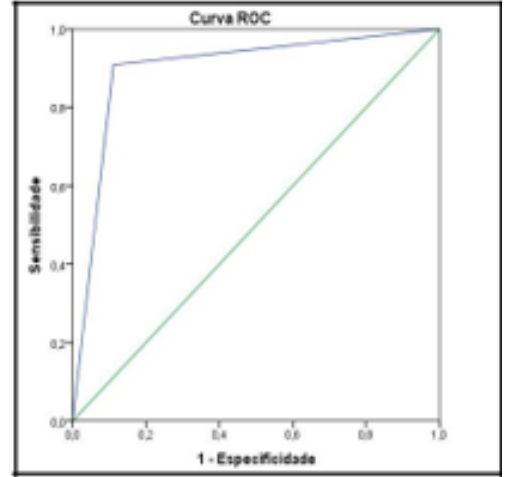

Figure 2: ROC curve for the CCTA in the analysis of CD by patient. Curva ROC $=$ ROC curve, Sensibilidade $=$ Sensitivity, Especificidade $=$ Specificity

The study proved to be very safe with only one minor skin allergic reaction, one vasovagal reaction and one subcutaneous infiltration of contrast agent due to extravascular extravasation being recorded as complications. No cases of contrast-induced nephropathy were recorded.

\section{Discussion}

Coronary computed tomography angiography using a SOMATOM Perspective $^{\diamond}$ scanner (Siemens AG) proved to be simple to carry out and the results of the coronary ICA, when compared with coronary angiography, were in line with the main reference studies using 64-detector scanners [1-3]. In the comparative analysis by patient, the high sensitivity and negative predictive value of the CCTA stood out, i.e. its robust capacity to exclude coronary disease, which is an aspect pointed out by several authors as its main advantage. The analysis by artery revealed a slightly lower sensitivity and a higher specificity (particularly in the RCA and Cx), which was slightly different from the usual finding and for which we could not find a valid explanation. The acuity in the LAD was in line with the majority of studies. The small sample may partly explain these findings.

This was a "real world" study in which the patients who underwent coronary angiography were selected based on the CCTA results and proposed for invasive study according to the opinion of the attending physician and the recommendation resulting from the final conclusions of the CCTA report. This fact may naturally create a bias in the selection of patients. However, we worked according to the "intention to diagnose" principle and considered that it would have been inappropriate, in the current state of knowledge, to carry out any other type of study (such as, for example, systematic assessment of all patients using both methods).

This methodology, despite being open to criticism, reflects current common clinical practice in terms of the diagnostic decision tree in most cases, i.e. the patient is referred for a CCTA due to a clinical or functional (non-invasive tests) suspicion of ischemia: if the CCTA does not reveal significant plaques or lesions, this diagnosis is excluded and the diagnostic process ends as the negative predictive value of this examination and its favorable prognostic value in terms of the risk of future cardiovascular events are well-known6. If the CCTA reveals suspected or definite lesions, the patient is proposed for coronary angiography and/or functional imaging tests (SPECT/Stress echocardiogram/stress MRI), depending on the practice and degree of suspicion as a result of the CCTA.

This diagnostic process model is recommended in the most recent guidelines, particularly by the National Institute of Clinical Excellence 
Pedro PG (2018) Use of coronary computed tomography angiography in the diagnosis and/or exclusion of coronary disease - Initial experience of one center and determination of the local acuity of the method, in comparison with coronary angiography

(NICE) [7] and in the myocardial revascularization recommendations of the European Society of Cardiology (ESC) at Level of Evidence A, Class IIa. This diagnostic strategy has recently been tested in major prospective and multi-center studies. In one of these the PROMISE study - the CCTA was no worse than traditional methods [9] and in another - the SCOT-HEART [10] study - it proved to be better, avoiding "clean" coronary angiographies. It is well-known that the use of coronary angiography in patients without significant coronary disease represents a significant cost for health services, as well as posing the risks and disadvantages inherent in the procedure. Some studies indicate that the cost of coronary angiographies where there is no significant disease is very high [11], partly due to inappropriate prescription, but also due to the low specificity of certain functional studies.

In this respect, the CCTA offers a precious advantage as it allows the selection, on an anatomical basis, of those patients who need to be sent for a coronary angiography, thus avoiding a significant number of unnecessary examinations [12].

Another advantage of the CCTA is the fact that, in patients with as yet non-obstructive coronary disease, it allows early atherosclerosis to be identified and opens an important therapeutic window in terms of "lifestyle" changes and intensive pharmacological intervention. This potentially allows the natural history of the coronary disease to be modified by stabilizing and even regressing the coronary plaque [13]. However, this theory has not yet been fully proven in large prospective studies, when compared with the conventional intervention strategies for traditional risk factors.

Despite this, in our opinion the identification of these nonobstructive plaques is essential because, as is well-known, it is these that are the most unstable and that will rupture and cause thrombosis, which is part of the physiopathological genesis of acute coronary syndromes [14]. The CCTA allows plaques to be detected non-invasively in the nonobstructive phase and in the future, thanks to the gradual improvement and development of the image quality, it will allow the identification of "vulnerable" plaques that are at higher risk of instability. Some studies are already pointing in this direction, having identified certain anatomical characteristics in addition to merely quantifying the coronary stenoses. These include the positive remodeling of plaque and the low density (in Hounsfield units) of the atheromatous deposits, resulting in a lipid core [15], spotty calcifications [16] and the "napkin-ring" sign, which is indicative of a thin endothelial layer coating atheromatous plaque [17].

Another very important area for the CCTA is the assessment of coronary artery bypasses [18]. This method is excellent at precisely characterizing the anatomy and patency of bypasses, which simplifies the study protocol for these patients and avoids exposure to multiple, unnecessary and technically more demanding coronary angiographies.

Areas that need to be further developed are the diagnosis of coronary stenoses in the presence of highly calcified plaques and the assessment of coronary stents, particularly small diameter stents $(<$ $3 \mathrm{~mm}$ ) as well as those that are placed over calcified plaques. In both situations, the presence of bright artifacts is a known limitation of the CCTA, which we hope to see overcome shortly with the introduction of technological improvements in the configuration of scanners, both through more accurate detectors and through ways of eliminating calcium artifacts, particularly using dual energy [19]. The incorporation of functional coronary information extracted from the CCTA, such as the coronary fractional flow reserve (CFFR) study [20] or CT coronary perfusion studies [21], is a rapidly expanding field that could transform the CCTA into a one-stop shop for cardiac and coronary anatomy and function, through a single examination.

\section{Conclusion}

Coronary CT angiography proved to be a simple, effective, noninvasive and safe method for assessing the coronary anatomy. In our study, conducted under local conditions, it proved to have a diagnostic capacity similar to that described in the main reference studies. This assessment is regarded as vital because it contributes to the credibility of the technique used in each center.

\section{References}

1. Miller JM, Rochitte CE, Dewey M, Arbab-Zadeh A, Niinuma H, et al. (2008) Diagnostic performance of coronary angiography by 64-row CT. $N$ Engl J Med 359: 2324-2336. [Crossref]

2. Budoff MJ (2008) Diagnostic performance of 64-multidetector row coronary computed tomographic angiography for evaluation of coronary artery stenosis in individuals without known coronary artery disease: results from the prospective multicenter ACCURACY trial. J Am Coll Cardiol 52: 1724-1732 [Crossref]

3. Meijboom WB (2008) Diagnostic accuracy of 64-slice computed tomography coronary angiography: a prospective, multicenter, multivendor study. J Am Coll Cardiol 52: 2135-2144 [Crossref]

4. Diamond GA, Forrester JS (1979) Analysis of probability as an aid in the clinical diagnosis of coronary-artery disease. $N$ Engl J Med 300: 1350-1358. [Crossref]

5. Leipsic J (2014) SCCT guidelines for the interpretation and reporting of coronary CT angiography: A report of the Society of Cardiovascular Computed Tomography Guidelines Committee. J Cardiovasc Comput Tomogr 8: 342-358 [Crossref]

6. Min JK (2011) Age- and Sex-Related Differences in All-Cause Mortality Risk Based on Coronary Computed Tomography Angiography Findings - Results From the International Multicenter CONFIRM (Coronary CT Angiography Evaluation for Clinical Outcomes: An International Multicenter Registry) of 23,854 Patients Without Known Coronary Artery Disease $J$ Am Coll Cardiol 58:849-860. [Crossref]

7. Guidance.nice.org.uk/cg95

8. Windecker S, Kolh P, Alfonso F, Collet JP, Cremer J, et al, (2014) 2014 ESC EACTS Guidelines on myocardial revascularization: The Task Force on Myocardia Revascularization of the European Society of Cardiology (ESC) and the European Association for Cardio-Thoracic Surgery (EACTS)Developed with the special contribution of the European Association of Percutaneous Cardiovascular Interventions (EAPCI) Eur Heart J 35: 2541-2619. [Crossref]

9. Douglas PS (2015) Outcomes of anatomical versus functional testing for coronary artery disease. $N$ Engl J Med 372(14):1291-300.

10. The SCOT-HEART investigators [2015] CT coronary angiography in patients with suspected angina due to coronary heart disease: an open-label, parallel-group, multicenter trial. Lancet 385: 2383-91 [Crossref]

11. Ohlow M et al. (2010) Is Elective Coronary Angiography Overused in Patients with Suspected Coronary Artery Disease? Future Cardiol 16: 886-895. [Crossref]

12. Hannan EL et al Appropriateness of Diagnostic Catheterization for Suspected Coronary Artery Disease in New York State Circ Cardiovasc Interv 7:19-27 [Crossref]

13. de Graaf FR (2013) Non-invasive computed tomography coronary angiography as a gatekeeper for invasive coronary angiography Int J Cardiovasc Imaging 29: 221-228. [Crossref]

14. Gao WQ, Feng QZ, Li YF, Li YX, Huang Y et al. (2014) Systematic study of the effects of lowering low-density lipoprotein-cholesterol on regression of coronary atherosclerotic plaques using intravascular ultrasound. BMC Cardiovasc Disord 14:60. [Crossref]

15. Falk E, Shah PK, Fuster V (1995) Coronary plaque disruption. Circulation 92: 657671. [Crossref]

16. Motoyama S (2009) Computed tomographic angiography characteristics of atherosclerotic plaques subsequently resulting in acute coronary syndrome, J Am Coll Cardiol. 54: 49-57. [Crossref]

17. Puchner SB, Liu T, Mayrhofer T, Truong QA, Lee H, et al. (2014) High-risk plaque detected on coronary $\mathrm{CT}$ angiography predicts acute coronary syndromes independent of significant stenosis in acute chest pain: Results from the ROMICAT-II trial. $\mathrm{J} \mathrm{Am}$ Coll Cardiol 64: 684-692 [Crossref]

18. Seifarth H, Schlett CL, Nakano M, Otsuka F, Károlyi M, et al (2012) Histopathological correlates of the napkin-ring sign plaque in coronary $\mathrm{CT}$ angiography. Atherosclerosis 224: 90 - 96 [Crossref] 
Pedro PG (2018) Use of coronary computed tomography angiography in the diagnosis and/or exclusion of coronary disease - Initial experience of one center and determination of the local acuity of the method, in comparison with coronary angiography

19. Gabriel J (2015) Should computed tomography angiography supersede invasive coronary angiography for the evaluation of graft patency following coronary artery bypass graft surgery? Interact Cardiovasc Thorac Surg 21:231-9. [Crossref]

20. Fuchs TA (2014) Coronary artery calcium quantification from contrast enhanced CT using gemstone spectral imaging and material decomposition. Int J Cardiovasc Imaging 30:1399-1405. [Crossref]
21. Nørgaard BL (2014) Diagnostic performance of noninvasive fractional flow reserve derived from coronary computed tomography angiography in suspected coronary artery disease: the NXT trial (Analysis of Coronary Blood Flow Using CT Angiography: Next Steps). J Am Coll Cardiol. 63:1145-55. [Crossref]

22. Pelgrim GJ, The dream of a one-stop-shop: Meta-analysis on myocardial perfusion CT. Eur J Radiol 84: 2144-2420. [Crossref]

Copyright: $\odot 2018$ Pedro PG. This is an open-access article distributed under the terms of the Creative Commons Attribution License, which permits unrestricted use, distribution, and reproduction in any medium, provided the original author and source are credited. 